\title{
Ideologías lingüísticas en un centro de investigación en México
}

\section{Linguistic ideologies in a Mexican research center}

\author{
Colette Ilse Despagne BroXner* \\ ElizABETH SÁNCHEZ MARTÍNEZ**
}

El objetivo de este estudio de caso crítico es identificar si las ideologías lingüísticas de los docentes-investigadores que laboran en un centro de investigación en ciencias sociales y humanidades en México influyen en su quehacer académico. La metodología es de corte cualitativo y se utilizaron cuestionarios, entrevistas y documentos oficiales para triangular los datos recolectados y, así, aumentar la fiabilidad de los resultados. Estos muestran que, en lo referente a la producción académica, se ha establecido un monolingüismo del inglés a nivel global y un monolingüismo del español a nivel local, que tienen impacto en las creencias y actitudes de los participantes. Por lo mismo, hacemos un llamado a la comunidad científica para adoptar un enfoque multilingüístico en el campo de la producción académica en la educación superior, lo que podría posiblemente ayudar a mantener la diversidad lingüística mexicana.

This critical case study aims to analyze the linguistic ideologies of researchers working at a Mexican research center in social sciences and humanities, and whether these ideologies influence their academic work. To triangulate the collected data of this qualitative research, we used questionnaires, interviews and official documents to increase the reliability of the results. Findings show that, in terms of academic production, English monolingualism has been established at a global level and Spanish monolingualism at a local level, which clearly impacts on the beliefs and attitudes of the participants. Participants perceive English as a bridge, a lingua franca, an international language and as the only language which would allow their research to achieve global impact. We therefore want to make a call on the scientific community to adopt a more multilingual approach in the field of academic production in higher education, which could possibly help maintain Mexico's linguistic diversity.

\section{Palabras clave:}

ideologías lingüísticas, ecología lingüística, México

\section{Keywords:}

linguistic ideologies, language ecology, Mexico

Recibido: 19 de junio de 2020 | Aceptado para su publicación: 11 de noviembre de 2020 | Fecha de publicación: 11 de enero de 2021

Recuperado de: https://sinectica.iteso.mx/index.php/SINECTICA/article/view/1168 doi: 10.31391/S2007-7033(2021)0056-004

\footnotetext{
* Doctora en Educación. Profesora-investigadora en el Instituto de Ciencias Sociales y Humanidades Alfonso Vélez Pliego de la Benemérita Universidad Autónoma de Puebla. Líneas de investigación: lingüística aplicada crítica, sociolingüística, educación intercultural, migración de retorno EUA-México. Correo electrónico: colette.despagne@gmail.com/https://orcid.org/0000-0001-9636-5685

** Maestra en Ciencias de Lenguaje por el Instituto de Ciencias Sociales y Humanidades Alfonso Vélez Pliego de la Benemérita Universidad Autónoma de Puebla. Líneas de investigación: lingüística aplicada crítica, sociolingüística, pragmática. Correo electrónico: sanmartelizabeth@gmail.com
} 


\section{INTRODUCCIÓN}

T a reflexión de este artículo versa sobre las ideologías lingüísticas que han influido en la difusión y valoración de unas lenguas y en la desaparición de otras. En el mundo, en los últimos diez años, han desaparecido más de 100 lenguas, principalmente indígenas, otras 400 están en situación crítica y 51 son habladas por una sola persona. Cada 14 días se muere un idioma de los casi 7,000 existentes (Unesco, 2003). Una lengua está en peligro de extinción cuando sus hablantes dejan de usarla, cuando se utiliza en un número cada vez más reducido de ámbitos de comunicación y, sobre todo, cuando ya no se enseña a las nuevas generaciones. Las razones son múltiples y pueden ser el resultado de fuerzas tanto externas, como la imposición de una lengua por razones económicas, religiosas o educativas, como internas, como la actitud negativa hacia ciertas lenguas y la sobrevaloración de otras.

En general, las presiones internas tienen su origen en las presiones externas (Unesco, 2003). En México, el español ha sido impuesto y hoy es la única lengua de prestigio usada en todos los ámbitos comunicativos, a pesar de que existen otras 68 lenguas indígenas, subdivididas en 364 variantes lingüísticas. Sin embargo, las lenguas indígenas están desvaloradas en el ámbito nacional, aunque la Ley General de Derechos Lingüísticos de los Pueblos Indígenas las declaró parte del patrimonio cultural y lingüístico y expresión de la composición pluricultural de la nación mexicana (INALI, 2009). Las fuerzas sociohistóricas externas impusieron un lingüicidio (Skutnabb-Kangas, 2012), o genocidio lingüístico, al otorgar al español el estatus de única lengua nacional de facto y al minorizar a las lenguas indígenas.

Ante este panorama, se prevé la disminución alarmante de la diversidad lingüística mundial (Unesco, 2003), y de la mexicana en específico: de las 364 variantes lingüísticas, 64 están en peligro muy alto de desaparecer, 43 alto, 3 mediano y 185 no están en un riesgo inmediato de desaparecer (INALI, 2009).

Skutnabb-Kangas (2011) considera a la educación como uno de los principales impulsores del lingüicidio, pues a través de ella se transmiten creencias y actitudes negativas; no obstante, también sostiene la posibilidad de contribuir a la diversidad lingüística desde ese ámbito. Este trabajo concuerda con la postura de SkutnabbKangas, aunque no se estima el único medio ni tampoco el más sencillo. Por lo mismo, nos fijamos el objetivo, en este artículo, de analizar las ideologías lingüísticas, entendidas como las creencias y actitudes hacia las lenguas, y su influencia en el quehacer académico de docentes-investigadores pertenecientes a un centro de estudios de educación superior en México, que en este trabajo renombramos Centro Público de Investigación sobre Estudios Sociales (CPIES).

Específicamente, nos propusimos identificar las creencias de diez docentes-investigadores del CPIES respecto a las lenguas nacionales y extranjeras y saber cómo estas creencias influyen en sus actividades de docencia e investigación. Esto es de suma importancia, puesto que las ideologías reproducidas en los entornos educativos pueden favorecer o desfavorecer a ciertas lenguas, y tener un impacto en la diversidad lingüística nacional y, por lo tanto, mundial.

Para cumplir con el objetivo propuesto, en este artículo referimos, en primer lugar, cómo la lingüística aplicada crítica puede contribuir, desde un punto de vista teórico, a analizar las ideologías lingüísticas de un contexto ecológico específico. 
En segundo lugar, nos enfocamos en el análisis de los contextos macro- y micropara entender las fuerzas externas que llevan a ciertas creencias y actitudes y, luego, especificamos la metodología de corte cualitativo que usamos en este trabajo. Finalmente, presentamos los resultados antes de concluir con una discusión sobre cómo los participantes en este estudio reproducen y, a la vez, resisten las ideologías lingüísticas dominantes del contexto mexicano.

\section{ECología E IDEOLOGíAS LINGÜÍSTICAS}

Este trabajo se inscribe en el área de la lingüística aplicada crítica y se fundamenta en las teorías sobre ecologías e ideologías lingüísticas. Dicha lingüística defiende la unión entre teoría y práctica, la actitud crítica sobre las desigualdades sociales, políticas y de poder expresadas y perpetuadas a través de la lengua (Pennycook, 2001; Cook, 2003). En ella se entrelazan temas referentes al poder, resistencia, derechos lingüísticos, conciencia crítica de las lenguas, ecologías e ideologías lingüísticas, y se recurre al análisis de los contextos macro- y micro- de la situación estudiada.

Lo anterior también es relevante para la ecología lingüística, pues presupone la relación tripartita entre lengua, hablantes y su contexto, social o geográfico (Haugen, 2001; Skutnabb-Kangas, 2011; Wendel, 2005). Dicha relación se manifiesta, a su vez, en las creencias y actitudes de los hablantes, es decir, en sus ideologías, así como en los discursos y las prácticas a nivel estatal, institucional, nacional y global (Blackledge, 2008). Por lo tanto, para un análisis de las ideologías en torno a las lenguas nacionales y extranjeras imperantes en el CPIES es necesario integrar un enfoque ecolingüístico que definimos a continuación.

\section{Ecología lingüística}

La ecología lingüística de algunas regiones era diversa (Haugen, 2001), pero se vio amenazada por cambios sociohistóricos, como la creación de Estados-naciones basados en la propagación de una sola lengua y una sola identidad. El resultado fue el paso acelerado del multilingüismo al monolingüismo, pues los mecanismos de supervivencia de las lenguas minorizadas son escasos y tienden a extinguirse. Por causa del lingüicidio (Skutnabb-Kangas, 2012), también llamado glotofagia (Calvet, 2002), es decir, del proceso político-social mediante el cual las lenguas desaparecen parcial o totalmente, víctimas de una lengua y una cultura dominante, la diversidad lingüística ha ido disminuyendo, incluso más rápido que la diversidad biológica.

Apelar a la diversidad lingüística significa que los idiomas y las culturas son igualmente necesarios para el equilibrio del planeta, tanto como lo biológico (SkutnabbKangas, 2012). Por lo tanto, los Estados-naciones, la globalización, la educación, los gobiernos y las élites son los principales responsables, y las poblaciones indígenas y las minorías lingüísticas son las más afectadas. Una vía para restablecer la diversidad son los derechos humanos lingüísticos. Por ello, se propone que el aprendizaje de lenguas sea aditivo, no sustractivo, es decir, que las lenguas maternas no se pierdan al aprender otras. Skutnabb-Kangas $(2001,2011)$ apuesta por el multilingüismo como medio para salvaguardar la diversidad y los derechos lingüísticos. 
De las 7,000 lenguas que existen en el mundo, alrededor de 250 son habladas por el 97\% de los habitantes. Así, sobre el 3\% de la población recae la diversidad lingüística mundial (Unesco, 2003). Ante tales cifras se estima que, al finalizar nuestro siglo, al menos 5,000 lenguas del mundo desaparecerán y quedarán en su lugar las lenguas dominantes. De acuerdo con el sistema lingüístico social de Swaan (2002), las lenguas hipercentrales y supercentrales corren menor riesgo de morir.

El inglés es la lengua hipercentral por excelencia de nuestra era, ya que ha sido posicionada como lengua global, internacional y franca. Las lenguas supercentrales, como el francés, español o alemán, son ampliamente habladas y sirven como conectores entre las lenguas centrales; las lenguas nacionales o centrales, como el holandés, el danés o el sueco, son las lenguas oficiales de un determinado territorio y son habladas por sus poblaciones. Por su parte, las lenguas regionales o vernáculas, como las lenguas indígenas de México, con menor número de hablantes y menor poder, son, por ende, las más amenazadas. Esta jerarquización de las lenguas es producto de relaciones sociohistóricas de poder, tanto a nivel global como local, y deriva en ideologías lingüísticas, es decir, en representaciones culturales, explícitas o implícitas, que definiremos en seguida.

\section{Ideologías e ideologías lingüísticas}

Las ideologías, percibidas como un sistema de ideas básicas compartidas con un grupo social, se expresan y reproducen a menudo por medio del lenguaje; las ideologías lingüísticas son representaciones mentales o ideas básicas que articulan nociones sobre las lenguas junto con las dimensiones sociales, culturales o políticas (Woolard, 2021), de acuerdo con los intereses de un grupo social específico (Kroskity, 2004). Van Dijk (2006) no se concentra en las ideologías lingüísticas en particular, pero precisa que el concepto de ideología se refiere a un conjunto de creencias fundamentales, socialmente compartidas, que guían o controlan prácticas, y comprenden tres ejes: el cognitivo, creencias en cuanto pensamiento; el social, creencias asociadas con intereses, conflictos, luchas de grupos; y el discurso, como medio de reproducción de las ideologías, mediante el sistema educativo, por ejemplo.

Con base en esta orientación, entendemos las ideologías lingüísticas como aquellas creencias fundamentales sobre las lenguas, socialmente compartidas, que han guiado o controlado prácticas en torno a ellas. Las ideologías son aprendidas, reproducidas o resistidas a través del discurso (Van Dijk, 2006). Algunas ideologías son aprendidas o adquiridas de manera más explícita y, en consecuencia, "desde arriba”, como la religión, la política o la metodología científica. Otras ideologías se integran de manera implícita en la vida cotidiana y se reflejan en cuestiones de género, de raza o clase, aunque estas últimas se hacen explícitas cuando hay conflicto, lucha o resistencia (Van Dijk, 2001).

Las ideologías lingüísticas se integran como creencias sobre los idiomas de un cierto contexto y están estrechamente ligadas a las creencias sobre sus hablantes. Ayudan a explicar por qué ciertos idiomas juegan el papel que tienen "en la producción y la reproducción del orden social y del orden moral que lo legitima" (Heller, 2010 , p. 102). Por lo tanto, estas creencias pueden ser percibidas como neutrales o críticas, dependiendo de la afiliación de los autores. Si adoptamos un enfoque ecológico de lenguas, debemos también analizar las ideologías sobre el inglés, una lengua 
muy presente en el contexto académico mexicano. Las ideologías hacia esta lengua se mueven dentro de un continuo más o menos crítico.

La primera posición percibe al inglés como un fenómeno natural y su propagación es vista como algo bueno para la humanidad. Los adeptos de este enfoque liberal (De Swaan, 2002; Van Parijs, 2011) piensan que este idioma es superior a los demás por su naturaleza y su función, que sirve como lingua franca y, por ende, como medio de la comunicación internacional; su éxito es el resultado del imperialismo británico y de la fuerte influencia de la economía de Estados Unidos. Sin embargo, piensan también que el inglés, como lengua hipercentral, sirve como instrumento para fomentar democracia y progreso. Tanto De Swaan como Van Parijs rechazan la existencia de un vínculo entre idioma y cultura y, por lo mismo, piensan que la cultura puede ser expresada a través de cualquier idioma. Para ellos, adoptar el inglés como lengua de comunicación intercultural no significa acoger los valores y la visión cultural de esta lengua.

La segunda ideología, una visión mucho más crítica, hace hincapié en los riesgos de adoptar un solo idioma, específicamente el inglés, y se centra en las implicaciones políticas en relación con la ecología lingüística, el imperialismo lingüístico (Phillipson, 2009), los derechos lingüísticos (Skutnabb, 2001), y el papel del inglés en contextos poscoloniales. Grin (2005) analiza las repercusiones económicas negativas de la adopción mundial del inglés en los no angloparlantes, mientras que Ives (2006) pone de relieve la necesidad de tomar en consideración los valores simbólicos transmitidos a través de los idiomas, y en especial del inglés.

Esta visión crítica percibe, por lo tanto, que la adopción del inglés no es una libre elección basada de manera exclusiva en consideraciones racionales. En México, por ejemplo, el inglés es de hecho la única lengua extranjera enseñada en las aulas; los alumnos no escogen la lengua que quieren aprender. Por lo mismo, los adeptos de esta ideología abogan por el multilingüismo, y el desarrollo de una competencia multilingüe en las escuelas, como la única forma viable de impedir que los valores y la cultura angloamericana sean impuestos junto con el uso del inglés. Desde esta perspectiva crítica, el inglés es deseable solo para fines específicos.

La tercera ideología, más intermedia, en relación con la propagación del inglés, es la que apoyan Crystal $(2000,2012)$ y Graddol (2006). Su enfoque es diametralmente opuesto al de De Swaan, ya que adoptan una visión de ecología lingüística. Esta ideología intermedia se parece a la segunda ideología porque ambas favorecen el multilingüismo y creen que los idiomas definen una visión específica del mundo y un sistema único de conocimiento que debe mantenerse. La desaparición de un idioma representa para ellos una pérdida irremediable para la humanidad, porque con este se pierde toda una cultura y un sistema de valores. Sin embargo, en oposición a la tercera ideología, la segunda está enraizada en teorías críticas que analizan las relaciones de poder y efectos culturales y simbólicos de las lenguas.

Crystal y Graddol reconocen que la supremacía del inglés es la consecuencia del poder y el prestigio del cual gozan las naciones que lo hablan, aunque Crystal (2012) piensa que es una "realidad evidente" que ahora es independiente de cualquier control social. Esta perspectiva también cree que el inglés no solo "pertenece" a las potencias occidentales. Muchas otras naciones expresan sus identidades culturales a 
través del inglés, lo que se puede observar en escritos poscoloniales, en artículos académicos y en narraciones de refugiados (Shemak, 2013).

Las voces en estas narraciones, o investigaciones, expresan su propia visión específica del mundo a través del uso del idioma inglés. Desde esta tercera perspectiva intermedia, el inglés tiene que ser aceptado como lengua franca y la diversidad lingüística tiene que ser salvaguardada. En México, esta diversidad ha vivido un largo proceso sociohistórico que nos puede proporcionar pistas para comprender el estado actual de las lenguas existentes en el territorio.

\section{SitUACIón LINGÜÍSTICA EN MÉXICo}

En México, más que una situación de diglosia, se vive un real estado de lingüicidio (Skutnabb-Kangas, 2001); en doscientos años, la población mexicana hablante de una lengua indígena disminuyó del 60\% al 7\% (Pastrana, 2012). Esto se debe, en gran medida, a los procesos de asimilación lingüística y cultural desarrollados desde la creación del Estado-nación mexicano en el siglo XX que han favorecido la homogeneización de su población sobre la base de una identidad única, la mestiza, y una lengua única, el español.

Dichos procesos conllevan una importante discriminación basada en la lengua, ya que estas políticas lingüísticas y educativas públicas han sido y siguen siendo claramente monolingües y monoculturales. Para tal efecto, la educación, mediante los procesos de castellanización, fue una herramienta extremadamente útil (Martínez, 2015). Como señala Aguilar (2019), "las lenguas indígenas no mueren. El Estado mexicano es quien las mata". Por lo mismo, el español es la única lengua oficial de facto que se usa en todos los ámbitos comunicativos en México, incluso en la educación. Las lenguas indígenas casi no se enseñan de manera formal, solo en el subsistema de Educación Intercultural Bilingüe de la Secretaría de Educación Pública, destinado de manera exclusiva a poblaciones indígenas y cuyos resultados son limitados (Schmelkes, 2013). La escasa valoración y enseñanza de lenguas indígenas en México se debe, en cierta medida, a las ideologías en torno a las lenguas, alimentadas por mecanismos de poder como el racismo y el lingüicismo, es decir, la discriminación negativa de las lenguas (Terborg y García, 2011).

A partir de 1994, la figura del inglés en el territorio nacional aumentó drásticamente, junto con la firma del Tratado de Libre Comercio entre México, Estados Unidos y Canadá. Este tratado impactó a nivel cultural y lingüístico (Despagne, 2015) y, desde este entonces, el rol de esta lengua en México es particular. Sin tener una presencia oficial, es cada vez más importante como lengua hipercentral y, por lo tanto, presente en la educación. El inglés es obligatorio en secundaria y bachillerato desde los años setenta y se han hecho varios intentos, aunque con resultados limitados, de incorporar su enseñanza en el currículo de la enseñanza básica desde la década de los noventa (Sayer, 2015), mientras que la enseñanza de lenguas indígenas es casi inexistente.

La Compañía de Recursos Humanos OCC Worldwide estimó que el 90\% de las ofertas de trabajo administrativo y educativo en México requieren un alto nivel de inglés, y que tener un diploma de este idioma como lengua extranjera aumenta las 
posibilidades de conseguir un trabajo en un 44\% (Universia México, 2014). Heredia y Rubio (2015) han demostrado una clara correlación entre el nivel de dominio del inglés y la situación socioeconómica en México. Por lo tanto, el inglés en México está vinculado a la movilidad social y, aunque no siempre se utilice en el trabajo, también se usa para descartar posibles candidatos.

De la misma forma, el inglés se ha vuelto el centro de los programas de internacionalización de las universidades (Despagne, 2019). Por consiguiente, se ha adoptado su enseñanza a nivel nacional, aunque los resultados todavía parecen muy limitados. En efecto, México se encuentra en un nivel "bajo" según el Índice de Competencia de Inglés, un índice internacional que evalúa la competencia lingüística en inglés como lengua extranjera de 100 países en el mundo (Education First, 2020). Del total de países evaluados a escala mundial, México se encuentra en el lugar 67, y de los 20 países latinoamericanos, en el número 17.

Aunado a esto, la lengua inglesa ocupa un alto porcentaje respecto a producción académica internacional. En 2000, las publicaciones en ciencias sociales y naturales fueron en inglés, en un 82\% y 90-95\%, respectivamente (Hamel, 2013), mientras que en 2010 alcanzaron un 94\% y 96\% en revistas internacionales indexadas (García, Alonso y Jiménez, 2013). En efecto, el inglés ha desplazado a otras lenguas y ha establecido un dominio en las ciencias y la educación superior que comienza con el control de las publicaciones científicas también en México.

De acuerdo con Montgomery (2013), el inglés se volvió la lengua franca moderna de la ciencia y en la circulación científica nacional, además de tener un monopolio casi total en el área de ciencias formales y naturales. Existe una hegemonía del inglés, pero sin un predominio total, en las ciencias naturales aplicadas y tecnologías. Mientras que en las ciencias sociales es visible la fuerte presencia del inglés, existen todavía amplios espacios propios en las lenguas nacionales (Hamel, 2013).

Las universidades se enrolan en esta mecánica para competir por el financiamiento de la investigación y por una posición dentro del mercado de los centros de educación superior (Hamel, 2013, 2017). Prueba de esto es la reciente convocatoria Ciencia Frontera 2019, del Consejo Nacional de Ciencia y Tecnología (Conacyt, 2019), cuyo objetivo es generar conocimiento novedoso en México con referentes de calidad internacional, que requirió que se enviaran todas las propuestas en inglés. Por ende, los investigadores que no podían redactar propuestas académicas en inglés quedaron excluidos.

Además, los docentes-investigadores, en el ámbito nacional, y específicamente los del CPIES, para ingresar o permanecer en una institución de educación superior, como la Universidad Autónoma (UA) o el Sistema Nacional de Investigadores (SNI), deben cubrir determinados requerimientos, entre ellos, la producción de artículos de investigación publicados en revistas de arbitraje riguroso, por lo general vinculadas a bases de datos reconocidas a escala internacional.

\section{Centro Público de Investigación sobre Estudios Sociales}

Actualmente, los centros o institutos de educación superior en México están atravesados por el discurso de la internacionalización. Este proceso refiere dos movimientos: 
el interno, dado desde la misma universidad y dirigido a mejorar la calidad en las investigaciones, docentes e investigadores, al adoptar métodos y procesos de gestión internacionales. El externo alude a la percepción de las universidades desde fuera (Sebastián, 2005). Por esta razón, los programas de internacionalización buscan dar mayor visibilidad y reconocimiento.

La universidad a la que está adscrito el CPIES creó un comité especializado en la internacionalización. El objetivo es identificar e impulsar líderes de grupos de investigación para una producción científica de calidad, con una carrera en el campo de la ciencia que impacte a nivel internacional. Los estándares para cumplir con estas metas se basan en los descriptores definidos por EURAXESS (European Commission, 2011), una iniciativa europea que apoya la movilidad de investigadores y el desarrollo internacional de sus carreras.

Dicha iniciativa delinea los perfiles deseables de los investigadores, de acuerdo con su experiencia y consolidación, divididos en cuatro niveles. Los niveles R4 no son similares a los del SNI, pues no implican un nombramiento o remuneración ni la antigüedad en un centro o instituto de investigación. No obstante, es requisito contribuir a la investigación de forma original, lo cual depende del área de conocimiento. Las ciencias naturales exigirán como resultados alguna patente y el área de tecnología, propuestas de innovación, creativas, pero el área de ciencias sociales y humanidades, por lo regular, se ciñe a dar resultados a través de las publicaciones nacionales e internacionales en revistas indexadas y arbitradas.

Estas últimas se consideran de alta calidad cuando se incluyen en catálogos, repertorios o bases de datos de alcance mundial, liderados por unos cuantos conglomerados, como Thomson-Reuters y Elsevier, a través de la Web of Science (WoS) o Scopus. En general, se ha naturalizado el English only (Hamel, 2017) en el círculo científico y en los organismos encargados de promover la investigación, innovación y desarrollo científico y tecnológico, así como en los centros de estudios superiores, como el CPIES, apegados a las demandas de aquellos.

El CPIES sigue una estrategia de internacionalización enfocada en diversificar y consolidar los programas de posgrado. En cuanto a consolidación, casi la totalidad de sus programas se encuentran inscritos en el Programa Nacional de Posgrados de Calidad. Respecto a diversificación, el CPIES es incluyente; cuenta con 250 estudiantes: 116 en el nivel de maestría y 134 en doctorado. Para dar mayor visibilidad al exterior, casi el 50\% de los alumnos presentaron avances de sus investigaciones en foros dentro y fuera del país. En 2018, profesores externos intervinieron en diversos coloquios, conferencias, presentación de libros y procesos de titulación, y maniobraron un diálogo de saberes y experiencias con otros centros de investigación a nivel internacional. Otra estrategia de diversificación son las estancias académicas. Por este motivo, el repertorio comunicativo de sus investigadores es amplio, al comprender lenguas hipercentrales, como el inglés, y supercentrales, como el español, principalmente.

\section{Metodología}

Este trabajo es un estudio de caso crítico, porque explora un fenómeno de la vida real (Yin, 2014) e integra la relación entre el macrocontexto sociohistórico y el 
microcontexto académico de los participantes. Las lenguas velan relaciones de poder, convenciones y prácticas establecidas (Taylor, Despagne \& Farahnaz, 2017) que se podrían ordenar y reordenar (Madison, 2012). De este modo, se involucra a los docentes-investigadores en lo concerniente a las ideologías lingüísticas en el entorno académico, fundamental para una perspectiva crítica.

Contamos con la participación de diez docentes investigadores activos, nacionales y extranjeros, cuyo grado máximo es de doctorado. Nueve de ellos son miembros del SNI, nivel I o II. Mediante los cinco niveles del SNI (candidato, nivel I, II, III y emérito), el Conacyt reconoce la labor de producir conocimiento científico y tecnológico a través de un nombramiento y estímulo económico. Los instrumentos utilizados para la recolección de datos estuvieron constituidos por un cuestionario y entrevistas a los docentes-investigadores, así como documentos oficiales.

En el cuestionario se indagaron características generales de los participantes: género, edad, nacionalidad, grado académico, lenguas habladas (nacionales o extranjeras) y nivel de competencia lingüística. Investigamos qué lenguas utilizaban en el ámbito académico y si consideran la existencia de una que posibilitara mayor difusión a sus investigaciones. A partir de la información recopilada, realizamos una entrevista semidirigida con cada uno de los participantes, y profundizamos en los datos obtenidos, al ligarlos con temas sobre identidad, diversidad y muerte de lenguas, así como el papel de las instituciones respecto a los dos últimos temas.

Los documentos oficiales y la transcripción de las entrevistas fueron tratados desde el análisis crítico del discurso, con el objetivo de dilucidar las expresiones ideológicas de las instituciones y los docentes-investigadores participantes. De acuerdo con la propuesta de Van Dijk (2005), utilizamos cuatro categorías básicas para examinar un discurso ideológico:

-Contexto: los participantes hablan como miembros de un grupo.

-Texto o discurso: presentación positiva de nosotros, identificados como miembros de un grupo, contra una presentación negativa del otro. En nuestro caso, consideramos también la presentación positiva o negativa respecto a las lenguas, y pusimos atención en los aspectos lingüísticos que resaltaban lo positivo o lo negativo en torno a ellas.

-Significado: comprende los temas o estructuras macrosemánticas, es decir, la información sobresaliente en cada discurso, que puede formularse de modo abstracto o específico, por medio de titulares, subtítulos o apartados. La información puede manifestarse implícita o explícitamente, precisa o vaga, al asumir o delegar responsabilidades (modalidad): se debe, debemos, se tiene.

-Forma: repetición de las elecciones léxicas.

Los documentos oficiales fueron las convocatorias para ingresar al SNI y al círculo de investigadores de la UA. Allí se buscó algún requisito para publicar en una lengua en particular. De acuerdo con las categorías de análisis, primero examinamos la adhesión a un grupo ideológico (comités evaluadores, profesores, investigadores); segundo, identificamos los temas o macroestructuras semánticas, es decir, la estructura de los textos, para enfocarnos en el apartado de evaluación de la producción científica; tercero, observamos si la información era explícita o implícita, 
vaga o precisa, además de la modalidad (interna o externa) para dirigirse a los aspirantes o miembros del SNI mediante reiteradas elecciones léxicas y determinar si todo esto influía en las creencias y actitudes de los investigadores. Expuesto de otro modo, nuestra intención fue advertir si existe una relación entre las lenguas habladas por los docentes-investigadores y los lineamientos de las convocatorias.

En lo que concierne al análisis de las entrevistas, buscamos convergencias y divergencias en los discursos de los participantes. A partir de las categorías señaladas, revisamos cómo los docentes-investigadores se identifican con su grupo ideológico y de qué manera. Analizamos las elecciones léxicas para determinar si sus posturas son explícitas, implícitas, vagas o precisas, así como la modalidad, es decir, en qué momento ellos asumen la responsabilidad de ciertas acciones y cuándo señalan a otros actores como los responsables. Nuestra atención se centró en los temas o macroestructuras semánticas, pues ahí se reagruparon las principales creencias y actitudes en torno a las lenguas, lo que hizo posible la discusión sobre la producción o reproducción de las ideologías lingüísticas.

Algunas de las limitaciones que este trabajo podría encontrar es la generalización apresurada con la que suelen acusar a los estudios de caso (Giménez, 2012). Sin embargo, para ello tendríamos que haber buscado generalizar y no fue el propósito de este estudio. Un estudio de caso se enfoca en un fenómeno de la vida real (Yin, 2014), lo que implica que ese fenómeno o situación tiene su propio contexto, manejado en este trabajo como microcontexto, con sus particularidades. Además, sumemos que el microcontexto, a su vez, está inserto en un macrocontexto.

Una manera de enriquecer la propuesta de la investigación quizá hubiera sido trabajar con una muestra que abarcara más participantes, porque quizá no parece representativa, al colaborar solo diez investigadores. También podrá ser más enriquecedor en futuras investigaciones considerar distintas áreas del conocimiento, pues por ahora nos orientamos al área de ciencias sociales y humanidades de un instituto de educación superior. Esto posibilitará una discusión más amplia, porque, como vimos, la presencia del inglés alcanza porcentajes más altos en la producción académica en las áreas de ciencias naturales.

A continuación, compartimos los resultados del análisis. Hemos omitido los nombres, así como información más detallada de los docentes-investigadores. De igual modo, utilizamos una clasificación simbólica (con números) para asegurarnos de guardar su confidencialidad.

\section{RESULTADOS}

En la convocatoria del Conacyt, la presentación de los miembros del grupo ideológico es positiva, destacan "sus cosas buenas" (Van Dijk, 2001) y se realiza a través de adjetivos como "honorable", "reconocida", personas con los "más altos niveles científicos, tecnológicos y humanísticos". No utiliza explícitamente el pronombre "nosotros", pero agrupa, por un lado, a los miembros del Conacyt en las categorías de "Comisiones Dictaminadoras", "Comisiones Dictaminadoras Pre-Evaluadoras" y "Comisiones Transversales", "evaluadores"; por otro lado, los académicos son reagrupados en las categorías "investigadores", "miembros" y "solicitantes" (para aquellos que todavía no 
son miembros, pero aspiran a serlo). Los propios participantes se identifican como profesores, académicos e investigadores. A veces, utilizan el adjetivo "uno" para hacer referencia a ellos como parte de un grupo: "uno como investigador" "nosotros tenemos que cumplir..., nosotros me refiero a los profesores jóvenes".

En las convocatorias del SNI y la UA prevalecen verbos modales, como "tener", "haber" y "deber". Esto nos indica una obligación externa, es decir, los lineamientos vienen de fuera; no dependen de los miembros o solicitantes, sino del Conacyt y UA; entonces, es necesario cumplirlos si se desea ser miembro. Esto es de particular interés, sobre todo en lo referente al tema o macroestructuras semánticas de "evaluación", encontrada en ambas convocatorias y que se enfoca en la producción científica: artículos, libros, capítulos de libros.

Para que la producción científica de los aspirantes a miembros del SNI o del círculo de investigadores de la UA obtenga una evaluación favorable, será necesario ceñirse a ciertos requisitos. Las elecciones léxicas más utilizadas por las convocatorias fue el adjetivo "riguroso" para referirse a los arbitrajes de las revistas de "prestigio" o "reconocidas", publicadas preferentemente en tres índices: WoS, Scopus y el Padrón de Revistas del Conacyt. Esto pareciera que no alumbra alguna manifestación explícita ni precisa sobre las ideologías lingüísticas; sin embargo, una revisión minuciosa arrojó la predilección por el inglés y el español.

\section{El inglés como lengua universal}

En la producción académica

Existe una relación estrecha entre las bases de datos mencionadas y las ideologías hacia las lenguas. El Padrón de Revistas del Conacyt integra revistas escritas en su mayoría en español, pero las otras dos bases de datos pertenecen o son editadas por los conglomerados angloparlantes Clarivate Analytics y Elsevier.

Clarivate Analytics, por ejemplo, a través de la colección de base de WoS, selecciona y registra 22,200 revistas y libros científicos en ciencias duras $(9,200)$, ciencias sociales $(3,400)$, y artes y humanidades $(1,800)$, en un total de 254 disciplinas (Web of Science Group, 2020); da una clara preferencia a las ciencias duras, a artículos en revistas y a publicaciones escritas en inglés. En 2010, las publicaciones en inglés en la Web of Science alcanzaban los siguientes porcentajes: 72\% en humanidades, 94\% en ciencias sociales y más del 96\% en ciencias naturales experimentales (Hamel, 2017). El español no alcanzó ni el 0.5\% (Hamel, 2013, 2017; García, Alonso y Jiménez, 2013).

Al comparar las publicaciones y las citas de los diez participantes en tres bases de datos, Google Scholar, Scopus y WoS, tuvimos los siguientes resultados: juntos contabilizaron 1,536 citas en Google Académico, mientras que Scopus arrojó 43 citas y WoS solo cuatro. Cabe destacar que nueve de los diez investigadores publican mayoritariamente en español, y en menor medida en francés, y solo un participante publica en inglés, el cual arrojó más citas en WoS y en Scopus. Los investigadores que no publican en inglés quedan invisibles en las bases de datos internacionales (Di Bitetti \& Ferreras, 2017), como Scopus y WoS, mientras que se les reconoce su producción académica en Google Scholar, una base de datos mucho más multilingüe que las dos anteriores. 
Vale la pena recalcar también que los investigadores en ciencias sociales en México publican muchos libros y capítulos de libros, lo que no se toma en cuenta ni en Scopus ni en el WoS. La ideología del inglés como "la lengua universal de la ciencia" (Hamel, 2017, p. 237) ya no es ningún secreto, en parte es implementada y apoyada por los conglomerados Clarivate Analytics y Elsevier, o Thomsons Reuters, todos angloparlantes. El Conacyt, al jerarquizar las bases de datos (las que más cuentan en las áreas sociales son Scopus y la WoS), parece legitimar un orden social (Heller, 2010) fomentado por estos conglomerados que se posicionan con claridad en un enfoque liberal (De Swaan, 2002; Van Parijs, 2011) que percibe la diversidad lingüística como un problema y al inglés como una lengua neutra de comunicación internacional desvinculada de cualquier tipo de intereses.

En las ideologías de los profesores

Los investigadores, en cierta forma, también reproducen este mismo orden social (Heller, 2010) al aceptar que, en los entornos académicos, así como en lo referente a producción académica, el inglés tiene una fuerte presencia que da más alcance a sus trabajos académicos. El I1, por ejemplo, afirma que "es básico tener algo en inglés, y si se puede en otro idioma, también es importante". Acepta que si desea tener mayor visibilidad a nivel global, puede recurrir a la traducción de sus trabajos al inglés, aunque dicha traducción no es siempre pagada por la institución.

Asimismo, la I2 sabe que algunos de sus trabajos han sido traducidos al inglés y añade que actualmente aquel "es el lenguaje oficial de los trabajos académicos". Esta ideología es compartida por el I3, quien sostiene que "a nivel mundial es la lengua académica [...] las revistas que están indexadas y valoradas como buenas métricas se publican en inglés". No obstante, él piensa que traducir sus trabajos al portugués podría llegar a otro público y no descartó realizar traducciones en lenguas indígenas; así, el I3 parece adoptar una visión más ecológica de lenguas al fomentar la relación tripartita entre lenguas, hablantes y contexto, mencionada por SkutnabbKangas (2011) y Wendel (2005).

El I4 añade que la presión externa provoca "que se publiquen muchas cosas en inglés"; sin embargo, él solo publica en español y francés. El I7, por su parte, también menciona que en los entornos académicos "la lengua oficial muchas veces es el inglés", y en relación con la asistencia a congresos, el público y el lugar, él presenta en español, francés o inglés. La I8 no ha publicado nada en inglés, pero afirma que este "es el idioma internacional". Para el I9, el inglés es "una lengua intermediaria entre muchísimas otras lenguas [...] porque es lo más accesible desde la educación formal”.

Los I4, I7, I8 e I9 perciben, por lo tanto, la imposición del inglés en la academia y que no es una libre elección (Phillipson, 2009; Skutnabb-Kangas, 2001), pero también advierten su supremacía como una "realidad evidente" (Crystal, 2012). La I10 la tilda, incluso, de "lengua universal":

Es un idioma que me comunica con amistades, con gente que conozco o sea que se hizo [...] más allá de una simple lengua para estudiar se volvió un lenguaje que puedo utilizar para comunicarme con gente que quiero y que de otra manera no sería posible comunicarnos, porque, por ejemplo, tengo amistades que son de Turquía entonces tampoco hablo turco, no hablo farsi, entonces es como lenguaje universal. 
La I5 confirma la ideología de la I10, al considerar que el inglés es una "lengua puente", cuya finalidad es eliminar la barrera del idioma y facilitar la comunicación; añade que "si ya sabes inglés, te puedes comunicar con gente de muchos otros países, aunque no sea su lengua materna". La I10 también menciona que el inglés es parte de su vida personal y profesional. Los temas que ella trabaja y su literatura son en inglés. Sus publicaciones son en inglés y sí aparecen en la WoS y en Scopus.

Para la I5 y la I10, el inglés, por ende, también expresa sus identidades y les pertenece (Shemak, 2013). Todos los investigadores coincidieron en la supremacía de la lengua en la academia y en el alcance que tienen, o tendrían, al publicar en inglés, pero, a pesar de las respuestas, no todos expresan con agrado lo anterior. Unos argumentan en contra de la simplicidad de la lengua como argumento para restarle importancia. Otros, en contra de la imposición de esta, producto del sistema neoliberal (Phillipson, 2009; Grin, 2005; Ives, 2006). El I1, por ejemplo, manifiesta:

La hegemonía cultural capitalista hace que, a partir de la Segunda Guerra Mundial, con este metarrelato del desarrollo, tenemos que hablar todo en inglés [...] el inglés es, es nuestra lengua oficial [...] todos tenemos que hablar en inglés, es una imposición cultural que tiene una ideología fuerte y una construcción también de uno que es la idea del desarrollo, es el American way of life que debe de llegar a todos lados y homologar.

Para la I6, todo se adecua a una cuestión de mercantilización:

Y hoy no vamos lejos, la misma universidad y todas nuestras instituciones nos piden certificaciones en las lenguas internacionales. [...] lo que interesa es lo que el mercado exige, lo que las políticas del Banco Mundial demandan, necesitamos gente que pueda acomodarse en los campos donde la internacionalización está mediada por el valor del dinero y las lenguas que tienen un gran prestigio para ser todos esos códigos comunicativos pues son las lenguas de prestigio económico, de prestigio histórico, de imposición y coloniaje en otros países, que pues claramente son el inglés, el francés y demás.

La mercantilización de las lenguas "internacionales" a la que se refiere I6 es, en efecto, fruto del capitalismo tardío que usan tanto a las lenguas como a las certificaciones lingüísticas como producto con valor de cambio en los procesos de internacionalización de las universidades y, en general, en la economía globalizada (Heller, 2010).

El I7, por su parte, pertenece a dos sociedades académicas en su área de conocimiento y pudimos percatarnos cómo el inglés fue impuesto como "lengua oficial". En su opinión, "no debe prevalecer ninguna lengua", en este caso "la dominante", cuyo estatus de dominación es dado "no por el número de personas que hablan esa lengua, sino por la situación económica y política”. La I8 concuerda con el I7, ya que lamenta que ni a ella ni a sus colegas del área de lingüística les valoran publicar en otras lenguas. Menciona "que deberían de apreciar más trabajos que están escritos en otras lenguas", distintas del inglés o del español.

Para el I4, el dominio del inglés en los entornos académicos "es un problema del colonialismo que sigue existiendo" y que posiciona al español y al inglés, por ejemplo, "como lenguas universitarias y como universales". La I2 considera que no se hace explícito, pero que sí se "valoran más los trabajos en inglés", con lo que tampoco está de acuerdo, pues es una "hegemonía que no se justifica". Según la I2, dicha hegemonía también tiene consecuencias en los "idiomas indígenas que son completamente marginados por otros idiomas" y, por ende, tienden a morir. 
Los I7, I8, I4 e I2 abogan todos por una visión claramente más ecológica y crítica de las lenguas que cuestiona las desigualdades expresadas y perpetuadas a través de estas (Pennycook, 2001; Cook, 2003) y, por ende, fomentan el multilingüismo al poner en relieve el riesgo que existe en adoptar el inglés como lengua única universal por las implicaciones políticas, económicas y neocoloniales que tiene (Phillipson, 2009; Skutnabb-Kangas, 2001; Grin, 2005; Ives, 2006).

En resumen, podemos observar que las ideologías lingüísticas más liberales de la UA y del Conacyt impactan fuertemente en las creencias y actitudes y, en consecuencia, en el quehacer académico de los docentes-investigadores que tienen que adecuarse al control social establecido. Si no publican en inglés, sus trabajos tienen menos alcance internacional y, por ende, serán menos citados en las bases de datos más reconocidas por ambas instituciones.

Como apunta la I10, los investigadores se vuelven más selectivos para publicar y buscan revistas en las que se van a reconocer sus trabajos. Se apegan a los lineamientos o, de lo contrario, "el esfuerzo realizado no se aprecia", como lo señala la I8, ya que, al no seguir la "realidad evidente" del inglés (Crystal, 2012) y al resistirse a la ideología liberal, tendrán muchas veces que renunciar a pertenecer al SNI o, incluso, a alguna institución. Una de las soluciones que encontraron los participantes en este trabajo para resistir, de algún modo, dentro del sistema establecido es publicar en español.

\section{El español como lengua "oficial" y de resistencia}

El español es lengua materna o nativa de cinco de los diez participantes en este estudio. En menor medida, figuraron otras lenguas, como el francés, alemán, portugués y solo uno de los investigadores habla otomí, totonaku y náhuatl.

Los docentes-investigadores destacaron el hecho de que el español es la lengua utilizada en su entorno académico. La I8 habla cinco lenguas; no obstante, gran parte de su vida personal y profesional la realiza en México; por lo mismo, utiliza más el español: "Me siento más cómoda, para concluir, exponiendo y escribiendo en español [...] prácticamente todos mis trabajos, los libros que he editado, todos están en español". La I6 también escribe en español, ya que trata temas sociales mexicanos.

El I7 apunta que su vida académica se desarrolla en México, por lo que básicamente utiliza el español, que para él "es fundamental". Para el I4, el español y el francés son sus lenguas de trabajo y publica en ambas. En la actualidad, está adscrito al CPIES y menciona que allí "el español es la lengua oficial con la que trabajamos". La I2, cuya lengua materna es el portugués, narró que, por cuestiones de migración, aprendió el español. Sus trabajos están en español. El I1 aprendió y utiliza el español por los temas de investigación en los que se enfoca; su "objeto de estudio" es Latinoamérica y sus publicaciones están en español y en italiano.

Además de ser una lengua fundamental y académica en la vida de los participantes, el español también es una lengua de resistencia para tres de ellos. El I3 comenta que las presentaciones en congresos, así como los seminarios y publicaciones, son en español. Para él, una manera de aportar a la diversidad lingüística es trabajar en la lengua propia. Al preguntarle sobre alguna forma de resistir a la muerte y desaparición de lenguas, responde que la resistencia radica en que "nosotros estemos hablando en español". 
En este mismo sentido, se encuentra la visión de la I5, cuyas investigaciones abordan la ecología política latinoamericana, por lo cual toda su producción académica, salvo una traducción al francés de uno de sus artículos, está en español. Ella es consciente, por un lado, del público al que van dirigidos sus estudios, el cual es sobre todo latinoamericano; por esta razón, no tiene la necesidad de utilizar otra lengua. Por otro, también sabe de la existencia de interlocutores de habla distinta al español, pero piensa que si hay interés "pues tienen que aprender español":

Yo trabajo cuestiones de ecología política latinoamericana, entonces muchos de mis interlocutores son latinoamericanos y de mis lectores también, entonces yo creo que como que no lo he planteado como una necesidad, que los temas que trabajo son muy de esta región y entiendo que los lectores más interesados son de esta región, aunque evidentemente habrá otros hablantes, de otro idioma que si les interesa pues tienen que aprender español ¿no?

La lengua española, por ende, como las lenguas originarias mexicanas, se vuelven lenguas de resistencia, cuyo objetivo es contrarrestar el poder y la imposición de la lengua dominante más cercana en la ecología lingüística local. Como lo menciona la I5, el uso del español en la academia puede compensar el poder del inglés, tal como el uso de las lenguas originarias mexicanas pueden equilibrar el poder del español impuesto por el Estado-nación mexicano.

El I9 tiene una creencia similar a la de la I5, es decir, que los demás deberían aprender español si les interesa su trabajo. Sus motivos están ligados, según su percepción, a la riqueza de la lengua española, su grado de complejidad y sus diversas variantes regionales, todos argumentos para enaltecer la superioridad de una lengua sobre otra (Phillipson, 2009): "Me he empecinado en utilizar el español porque es una lengua muy compleja, con muchísimas variantes regionales, incluso en el propio país, y como también me gusta escribir literatura, es una cosa que me gusta seguir explorando".

Como se puede apreciar en las citas mencionadas, existe todavía una fuerte tradición y presencia del español en ciencias sociales y humanidades (Hamel, 2017), tanto en el CPIES como en México, que se puede apreciar en la lista de revistas mexicanas avaladas por el Conacyt, pero no por la WoS ni por Scopus.

De los tres investigadores, solo el 19 expresó que la resistencia al utilizar el español es frente a la lengua inglesa: “... que los interesados en las investigaciones que uno realiza que las lean en español, que los angloparlantes busquen otra lengua”. No lo dice explícitamente, pero lo considera al sugerir que los "angloparlantes" deberían leer en otras lenguas distintas al inglés. Este idioma ya tiene un monopolio en la educación superior y en las publicaciones, y es que, con la imposición de una lengua, viene la imposición de discursos académicos, temas y modelos culturales de hacer ciencia (Hamel, 2017).

\section{DiSCUSIÓN Y CONCLUSIóN}

De acuerdo con Van Dijk (2006), las ideologías de los participantes en torno a las lenguas controlan sus prácticas: publican mayoritariamente en español, una lengua que perciben como lengua de resistencia frente al inglés, pero aceptan la "realidad evidente" del poder del inglés (Crystal, 2012) en la academia, aunque 
critican su hegemonía. Sin embargo, destaca también que los investigadores del CPIES están atrapados entre dos presiones lingüísticas y culturales que se resumen a continuación: la presión del inglés como producto de la globalización y de la internacionalización de las universidades, y la presión hegemónica del español como producto de la ideología del Estado-nación mexicano.

\section{Presión por el inglés}

Instituciones como la UA y el Conacyt legitiman una ideología lingüística liberal a favor del inglés (De Swaan, 2002; Van Parijs, 2011) al dar preferencia a los índices WoS y Scopus en la jerarquización de las publicaciones en las evaluaciones de los profesoresinvestigadores, lo cual ejerce presión sobre quienes no publican en inglés y legitima un orden social (Heller, 2010) que percibe al inglés como la única lengua franca de la academia (Hamel, 2017). Esta presión por parte de las instituciones, que representan el discurso dominante, tiene un impacto importante sobre las ideologías lingüísticas de los profesores-investigadores que posicionan al inglés como algo básico, el lenguaje oficial, la lengua académica de las buenas revistas, la lengua oficial e incluso universal, una lengua puente entre diferentes culturas y un idioma internacional.

A primera vista, estas ideologías podrían converger con la ideología liberal de De Swaan (2002) y Van Parijs (2011), que rechaza un vínculo entre lengua y cultura y que concibe al inglés como la lengua de la ciencia y la comunicación intercultural. Esta ideología también induce al pragmatismo de los participantes, ya que están convencidos de que, si publicaran en inglés, sus trabajos tendrían mucha más visibilidad a nivel internacional. Sin embargo, también podemos constatar que, en sus diferentes discursos, los participantes hacen hincapié en la visión colonial y neoliberal del inglés, lo que los lleva a adoptar una ideología crítica más acorde con las ideologías lingüísticas de Grin (2005), Ives (2006) y Phillipson (2009), quienes sostienen que el inglés no es una libre elección, pero que la tienen que usar específicamente en el ambiente académico.

Desde este enfoque, los participantes en este estudio también consideran al inglés como una lengua demasiado "sencilla" con la cual no pueden expresar lo mismo que en español, una "imposición cultural del neoliberalismo" que se basa en el "mercantilismo" y en las "políticas del Banco Mundial"; se refieren a la imposición del inglés, desde la percepción de la lengua del desarrollo (De Swaan, 2002; Van Parijs, 2011), como a un "colonialismo continuo", lo que nos demuestra que las ideologías lingüísticas de los participantes toman en cuenta los valores simbólicos transmitidos mediante la lengua (Ives, 2006). Por ende, difieren de las ideologías de Crystal (2012) y Graddol (2006), porque advierten un grado de control social a través del uso del inglés. Desde las ideologías lingüísticas de los participantes, lengua y cultura están estrechamente relacionadas. Esta visión crítica del inglés los lleva a percibir al español no solo como la lengua académica de todos, sino también como una lengua de resistencia.

\section{Presión por el español}

Los diez participantes señalaron al español como su lengua académica, aunque, para la mitad de ellos, el español no es su lengua nativa. Sin embargo, la mayoría realiza todas 
sus actividades académicas en esta lengua. Tres de ellos van más allá del español como lengua "oficial", y por lo mismo fundamental en sus vidas; califican al español, además, como una "lengua de resistencia" frente a la imposición del inglés en la academia. Su resistencia radica en que están hablando y escribiendo en español y no en inglés.

Este punto nos lleva a preguntarnos si existe en realidad una resistencia o una reproducción de las ideologías dominantes, "creadas desde arriba". Hay, en efecto, una resistencia frente al inglés, porque piden a los angloparlantes aprender español y publican principalmente en español; al mismo tiempo, reproducen las ideologías del español como lengua única, como "lengua académica", de "trabajo" y "lengua oficial".

Esta ideología parece beneficiar otra vez a la visión monolítica del Estado-nación mexicano basada en el monolingüismo del español (Despagne, 2015), junto con la idea de identidad única, inducida mediante el sistema de educación nacional (Martínez, 2015), ya mencionado. Lo anterior se destaca al encontrar a un solo investigador, de los diez participantes, como hablante o conocedor de lenguas indígenas. Los resultados muestran, entonces, que sí existe cierta resistencia de los docentes-investigadores frente a la hegemonía del inglés como lengua hipercentral en el ámbito académico, pero por medio del español, una lengua supercentral que se impone frente a las lenguas indígenas que no figuran para nada en la academia y que se siguen matando (Aguilar, 2019).

Con base en estos resultados, resaltamos la importancia del enfoque ecológico de las lenguas, que nos permite ver las tensiones entre todas las lenguas en un contexto específico. Si bien el uso del español puede representar una resistencia frente la imposición del English only (Hamel, 2017) en la academia, es necesario tomar en cuenta que, frente a las lenguas indígenas, el español también sigue siendo hegemónico. Por lo tanto, una visión multilingüística (Hamel, 2017; Ammon, 2012; SkutnabbKanguas, 2011; Phillipson, 2009; Creese \& Blackledge, 2011) como la expresada por los I7 e I8, que no se centre en las lenguas en aislamiento y que busque un aprendizaje aditivo de ellas, podría apoyar el mantenimiento e, incluso, el desarrollo de una parte de las lenguas indígenas en la educación superior en México.

Transitar hacia una orientación multilingüe significaría buscar modificar las ideologías lingüísticas de las instituciones, sean universidades, centros de investigación o instancias que gestionan la comunicación de la ciencia, a fin de transformar las creencias enraizadas de sus actores sociales y de las instituciones para lograr una convivencia más equilibrada (Hamel, 2008).

Transformar las ideologías lingüísticas no es tarea fácil, pero podría ser factible si se busca desarrollar una conciencia crítica frente a la utilización, el mantenimiento y la difusión de las lenguas (Taylor, Despagne \& Farahnaz, 2018) y desafiar, así, el orden naturalizado respecto a las prácticas, relaciones y construcción de significados, al potenciar nuevas epistemologías y cosmovisiones, y organizar movimientos en contra del dominio de algunas lenguas y no contra las lenguas per se.

Centros de investigación en ciencias sociales como el CPIES podrían ser un lugar idóneo para resistir "desde abajo" la imposición de lenguas hiper- y supercentrales al fomentar políticas lingüísticas dentro de un marco plural y plurilingüe que fomente la enseñanza-aprendizaje, la evaluación, la generación y la divulgación de conocimiento alrededor de cuatro ejes lingüísticos: español, lenguas indígenas locales, inglés y demás lenguas internacionales. 


\section{REFERENCIAS BIBLIOGRÁFICAS}

Aguilar Yásnaya, E. (2019). Discriminación lingüística y derechos de los pueblos indígenas en México. En M. León-Portilla. Ciclo de conferencias ¿Quiénes somos los mexicanos? Diálogos en la multiculturalidad. COLNAL e INALI, Ciudad de México, 21 de mayo. Recuperado de http://colnal.mx/events/ quienes-somos-los-mexicanos-dialogos-en-la-multiculturalidad-ciclo-deconferencias-1

Ammon, U. (2012). Linguistic inequality and its effects on participation in scientific discourse and on global knowledge accumulation -With a closer look at the problems of the second-rank language communities. Applied Linguistics Review, vol. III, núm. pp. 333-355. https://doi.org/10.1515/applirev-2012-0016

Blackledge, A. (2008). Language ecology and language ideology. En Nancy H. Hornberger (ed.). Encyclopedia of language and education. Suiza: Springer.

Conacyt (2019). Convocatoria 2019 para ingreso o permanencia en el SNI. Gobierno de México. Recuperado de https://www.conacyt.gob.mx/index.php/ el-conacyt/convocatorias-y-resultados-conacyt/convocatorias-sistema-nacional-de-investigadores-sni/convocatorias-abiertas-sni/ingreso-o-permanencia-sni/18907-conv-sni-19-1/file

Cook, G. (2003). Applied linguistics. UK: Oxford University Press.

Creese, A. \& Blackledge, A. (2011). Separate and flexible bilingualism in complementary schools: Multiple language practices in interrelationship. Journal of Pragmatics, vol, XLIII, núm. 5, pp. 1196-1208. https://doi.org/10.1016/j. pragma.2010.10.006

Crystal, D. (2012). A global language. En P. Seargeant \& J. Swann (eds.). English in the world: History, diversity, change (pp. 152-177). UK: The Open University \& Routledge.

Crystal, D. (2000). Language death. UK: Cambridge University Press.

Despagne, C. (2019). English, internationalization, and EFL teachers' ideologies in Mexico. Profile: Issues in Teachers' Professional Development, vol. 21, núm. 1, pp. 43-57. https://doi.org/10.15446/profile.v21n1.69239

Despagne, C. (2015). Modernidad, colonialidad y discriminación en la clase de inglés. Revista TRACE, 68. CEMCA, Centro de Estudios Mexicanos y Centroamericanos, Ministère des Affaires Étrangères et du Développement International, France. https://doi.org/10.22134/trace.68.2015.3

De Swaan, A. (2002). Words of the World: The global language system. UK: Cambridge University Press.

Di Bitetti, M. S. \& Ferreras, J. A. (2017). Publish (in English) or perish: The effect on citation rate of using languages other than English in scientific publications. Ambio, vol. 46, núm. 1, pp. 121-127. https://doi.org/10.1007/s13280016-0820-7

Education First (2020). EF EPI. EF English Proficiency Index. México. Recuperado de https://www.ef.com.mx/epi/

European Commission (2011). Towards a European framework for research careers. Recuperado de https://cdn5.euraxess.org/sites/default/files/policy_library/towards_a_european_ramework_for_research_careers_final.pdf

García Delgado, J. L., Alonso, J. A. y Jiménez, J. C. (2013). El español, lengua de comunicación científica. México: Fundación Telefónica. 
Giménez, G. (2012). El problema de la generalización en los estudios de caso. Cultura y Representaciones Sociales, año 7, núm. 12, pp. 40-62.

Graddol, D. (2006). English next. UK: British Council.

Grin, F. (2005). L'anglais comme lingua franca: questions de coût et d'équité. Commentaire sur l'article de Philippe van Parijs. Économie Publique, vol. 15, pp. 33-41. Recuperado de http://journals.openedition.org/economiepublique/31/ https://doi.org/10.4000/economiepublique.31

Hamel, R. E. (2017). Enfrentando las estrategias del imperio: hacia políticas del lenguaje en las ciencias y la educación superior en América Latina. En A. García Diniz, D. Araujo y L. Kaminski (eds.). Poéticas e políticas da linguagem em vias de descolonização. Brasil: Pedro \& João Editores. Recuperado de http:// hamel.com.mx/Archivos-Publicaciones/78.-Hamel-2017-Enfrentando-lasestrategias.pdf

Hamel, R. E. (2013). El campo de las ciencias y la educación superior entre el monopolio del inglés y el plurilingüismo: elementos para una política del lenguaje en América Latina. Trabalhos em Lingüística Aplicada, vol. LII, pp. 321384. https://dx.doi.org/10.1590/S0103-18132013000200008

Hamel, R. E. (2008). Plurilingual Latin America indigenous languages, immigrant languages, foreign languages-towards an integrated policy of language and education. En C. Helot \& A. M. de Mejía (eds.). Forging multilingual spaces. Integrated perspectives on majority and minority bilingual education. EUA: Multilingual Matters.

Haugen, E. (2001). The ecology of language. En A. Fill y P. Mühlhäusler (eds.). The ecolinguistics reader. Language, ecology, and environment. UK: Continuum.

Heller, M. (2010). The commodification of language. Annual Review of Anthropology, vol XXXIX, pp. 101-114. https://doi.org/10.1146/annurev. anthro.012809.104951

Heredia, B. y Rubio, D. (2015). Inglés y desigualdad social en México. En Mexicanos Primero (ed.). Sorry. El aprendizaje del inglés en México. Mexicanos Primero, Visión 2030 AC.

INALI (2009). Programa de Revitalización, Fortalecimiento y Desarrollo de las Lenguas Indígenas Nacionales 2008-2012. México: SEP.

Ives, P. (2006). Global English: Linguistic imperialism or practical lingua franca? Studies in Language and Capitalism, vol. I, pp. 121-142. Recuperado de http:// hdl.handle.net/10680/1254

Kroskity, P. V. (2004). Language ideologies. En A. Duranti (ed.). A companion to linguistic anthropology (pp. 496-517). Blackwell.

Martínez Buenabad, E. (2015). La educación intercultural y bilingüe (EIB) en México. ¿El camino hacia la construcción de una ciudadanía democrática? Relaciones, vol. CXLI, pp. 103-131. Recuperado de http://www.revistarelaciones.com/index.php/relaciones/article/view/92

Montgomery, S. L. (2013). Does science need a global language?: English and the future of research. Chicago \& London: The University of Chicago Press.

Pastrana Peláez, S. A. (2012). Desaparición de las lenguas indígenas. En F. González González, H. Santos Bautista, J. García Leyva, F. Mena Angelito y D. Cienfuegos Salgado (eds.). De la oralidad a la palabra escrita. Estudios sobre el rescate de las voces originarias en el sur de México (pp. 275-292). Ciudad de México: Colegio de Guerrero y Editora Laguna. 
Pennycook, A. (2001), Critical applied linguistics: A critical introduction. EUA: Lawrence Erlbaum Associates.

Phillipson, R. (2009). Linguistic imperialism continued. UK: Routledge.

Sayer, P. (2015). Expanding global language education in public primary schools: The National English Programme in Mexico- Language, Culture and Curriculum, vol. XXVIII, núm. 3, pp. 257-275. https://doi.org/10.1080/07908318.2 015.1102926

Schmelkes, S. (2013). Educación para un México intercultural- Sinéctica, Revista Electrónica de Educación, núm. 40, enero-junio, pp. 1-12. Recuperado de https://sinectica.iteso.mx/index.php/SINECTICA/article/view/48

Sebastián, J. (2005). La internacionalización de las universidades como estrategia para el desarrollo institucional. Innovación Educativa, vol. V, núm. 26.

Shemak, A. (2013). Postcolonial refugee narratives. En I. Ness (ed.). The Encyclopedia of Global Human Migration. EUA: John Wiley \& Sons. https://doi. org/10.1002/9781444351071.wbeghm422

Skutnabb-Kangas, T. (2012). Linguistic genocide in education or worldwide diversity and human rights. UK: Routledge.

Skutnabb-Kangas, T. (2011). Language ecology. En J. O. Östman y J. Verschueren (eds.). Pragmatics in practice. UK: John Benjamins Publishing Company.

Skutnabb-Kangas, T. (2001). Los derechos humanos y la educación multilingüe desde una perspectiva ecológica. Revista Educación, vol. CCCXXIII, pp. 99115. Recuperado de http://www.educacionyfp.gob.es/dam/jcr:2302cb5a17dc-4a62-b88c-fff421685baf/re32607-pdf.pdf

Taylor, S. K., Despagne, C. \& Farahnaz, F. (2018). Critical language awareness. En J. I. Liontas (ed.). The TESOL encyclopedia of English language teaching: Teaching speaking and pronunciation in TESOL. EUA: John Wiley \& Sons. https:// doi.org/10.1002/9781118784235.eelt0660

Terborg, R. y García Landa, L. (2011). Muerte y vitalidad de lenguas indígenas y las presiones sobre sus hablantes. Ciudad de México: UNAM.

Unesco (2003). Muerte y peligro de desaparición de las lenguas. Recuperado de: http://www.unesco.org/new/fileadmin/MULTIMEDIA/HQ/CLT/pdf/LVE_ Spanish_EDITED\%20FOR\%20PUBLICATION.pdf

Universia México (2014). Los conocimientos de inglés son necesarios en el 90\% de las vacantes. Sección Mercado Laboral. CNN Expansión, 21 de enero.

Van Dijk, T. (2006). Ideología. Una aproximación multidisciplinaria. México: Gedisa.

Van Dijk, T. (2005). Ideología y análisis del discurso. Utopía y Praxis Latinoamericana, vol. 29, pp. 9-36.

Van Dijk, T. (2001). La multidisciplinariedad del análisis crítico del discurso: un alegato en favor de la diversidad. En R. Wodak y M. Meyer (eds.) (2003). Métodos de análisis crítico del discurso. México: Gedisa.

Van Parijs, P. (2011). Linguistic justice for Europe and for the world. UK: Oxford University Press.

Web of Science Group (2020). Clarivate. Web of Science. Recuperado de https:// clarivate.com/webofsciencegroup/

Wendel, J. N. (2005). Notes on the ecology of language. Bunkyo University Academic Journal, vol. V, pp. 51-76. Recuperado de https://www.ubunkyo.ac.jp/ center/library/image/fsell2005_51-76.pdf. 
Woolard, K. A. (2021). Language ideologies. En J. M. Stanlaw (ed.). The International Encyclopedia of Linguistic Anthropology (pp. 1-21). EUA: John Wiley \& Sons. https://doi.org/10.1002/9781118786093.iela0217. Recuperado de https://onlinelibrary.wiley.com/doi/epdf/10.1002/9781118786093. iela0217

Yin, R. K. (2014). Case study research. Design and methods (5a. ed.). EUA: Sage Publications. 